\title{
Concentration and Solvency Effects on the Excess Amount and Surface Free Energy of a Colloidal Particle in a Solution of Nonadsorbing Polymer
}

\author{
Remco Tuinier*,† and Gerard J. Fleer \\ Forschungszentrum Jülich, Institut für Festkörperforschung, 52425 Jülich, Germany, and \\ Laboratory for Physical Chemistry and Colloid Science, Wageningen University, \\ 6703 HB Wageningen, The Netherlands \\ Received July 14, 2004; Revised Manuscript Received August 31, 2004
}

\begin{abstract}
Analytical expressions are derived for the polymer excess amount and the grand potential (surface free energy) of flat and spherical surfaces immersed in a solution of nonadsorbing polymer chains in the mean-field approximation. We start from a recent mean-field expression for the depletion thickness $\delta$ which takes into account not only the effect of the chain length $N$ but also that of the polymer concentration $\varphi_{\mathrm{b}}$ and the solvency $\chi$. Simple expressions are obtained for the interfacial properties at a colloidal surface, using both the adsorption method and the osmotic route. For a sphere of radius $a$, the excess amount can be separated into a planar contribution $\Gamma=-\varphi_{\mathrm{b}} \delta$ and a curvature correction $\Gamma_{\mathrm{c}}=$ $-\left(\pi^{2} / 12\right) \varphi_{\mathrm{b}} \delta_{\mathrm{c}}{ }^{2} / a$, where $\delta_{\mathrm{c}}$ is a "curvature thickness" which is close to (but smaller than) $\delta$. The grand potential has a planar contribution $\Omega=(2 / 9) \varphi_{\mathrm{b}} / \delta$ and a curvature part $\Omega_{\mathrm{c}}=(\pi / 18) \varphi_{\mathrm{b}} / a$. We test the results against numerical lattice computations, taking care that the boundary conditions in the continuum and lattice models are the same. We find good agreement up to a polymer segment volume fraction of $10 \%$, and even for more concentrated solutions our simple model is reasonable. For spherical geometry we propose a new equation for the segment concentration profile which excellently agrees with numerical lattice computations. The results can be used as a starting point for the pair interaction between colloidal particles in a solution containing nonadsorbing chains, which is discussed in the following paper.
\end{abstract}

\section{Introduction}

When polymer chains are depleted from the surface of a colloidal particle, the so-called depletion interaction leads to an effective attraction between the colloidal particles in a colloid-polymer dispersion. ${ }^{1}$ In many theoretical studies on polymer depletion, the chains were considered as ideal (noninteracting random coils) ${ }^{2,3}$ or even simplified further as dilute hard ${ }^{4}$ or freely overlapping spheres. ${ }^{5}$ In recent years depletion of (fully) interacting polymers in solution was studied using various theoretical methods, such as the polymer reference interaction site model (PRISM), ${ }^{6,7}$ osmotic equilibrium theory (sometimes called free volume theory), ${ }^{8}$ density functional theory, ${ }^{9}$ a Gaussian core model, ${ }^{10,11}$ and computer simulations. ${ }^{11,12}$ The influence of the solvent quality for the polymer chains on the depletion interaction and phase behavior has gained very little attention. Sear described solvency effects on the phase behavior of small spheres and long polymer chains (the so-called "protein" limit) within a mean-field approximation. ${ }^{13}$ Using the PRISM scheme, the depletion interaction for various effective "temperatures" was studied, ${ }^{14}$ and a comparison of the spinodals for the theta and athermal limits was made. ${ }^{15}$ Since most experimental polymer systems are in between the athermal and theta limits, a theory covering the entire range of solvencies is highly desirable, especially when it could also predict the depletion interaction and phase behavior for arbitrary polymer-colloid size ratios.

A very useful tool for the analysis of the properties of polymers near interfaces, of which the case of polymer

\footnotetext{
Institut für Festkörperforschung.

$\$$ Wageningen University.
}

depletion is a special case, is the numerical selfconsistent-field (SCF) lattice theory by Scheutjens and Fleer, ${ }^{16}$ which is based upon a recurrency relation that is a discrete version of the Edwards equation ${ }^{17}$ for polymer chains in a self-consistent field. With this SCF method it is possible to study many features of polymers at interfaces; ${ }^{18}$ unfortunately, this model gives only numerical results. For the case of polymer adsorption, Fleer et al. ${ }^{19}$ derived analytical approximations for the polymer profiles and, hence, for the adsorbed amount at a flat interface using the ground-state approximation to the Edwards equation. Recently, we used a similar ground-state approximation to derive a mean-field expression for the depletion profile and the depletion thickness near a flat wall for arbitrary polymer concentration, chain length, and solvency. ${ }^{20}$

In this article we apply this mean-field equation to calculate the excess adsorbed amount and grand potential of single particles in both flat and spherical geometries. In the next paper, ${ }^{21}$ we analyze the pair interaction between two flat walls and between two spheres immersed in a polymer solution. In both parts we explicitly address the effect of solvency and polymer concentration. We derive analytical expressions and test the results against numerical lattice computations. We intend to investigate the phase behavior of hard spheres in a mean-field polymer solution as a function of the solvent quality in a future paper.

\section{Flat Geometry}

2.1. Depletion Thickness. The thickness $\delta$ of the depletion zone next to a repulsive hard wall depends on the chain length $N$, the solvency (expressed in the Flory-Huggins parameter $\chi$ ), and the volume fraction 
$\varphi_{\mathrm{b}}$ of polymer in the bulk solution. In a previous paper, ${ }^{20}$ we proposed the relation $\delta^{-2}=\delta_{0}{ }^{-2}+\xi^{-2}$, where $\delta_{0}$ is the depletion thickness at infinite dilution, which depends only on the chain length $N$, and $\xi$ is a generalized (chain-length-independent) correlation length which approaches the mean-field limits $\xi \sim \varphi_{\mathrm{b}}{ }^{-1 / 2}$ in semidilute solutions in a good solvent and $\xi \sim \varphi_{\mathrm{b}}{ }^{-1}$ under semidilute theta conditions $(\chi=0.5)$. We concluded that this relation works excellently for $0<\chi<0.45$ (where the $\tanh ^{2}$ profile of eq 2.4 below is a good approximation) but gives a systematic overestimation of $\delta$ for (semidilute) theta solvency. This is due to a different groundstate solution for the semidilute profile in a $\Theta$ solvent (see Appendix 1), which gives a depletion thickness $\delta$ $=p \xi$, with $p=\sqrt{3 / 2} \operatorname{atanh}(1 / \sqrt{3}) \approx 0.81 .{ }^{20}$ To account for this systematic deviation under theta conditions, we generalize our previous equation to

$$
\begin{gathered}
\frac{1}{\delta^{2}}=\frac{1}{\delta_{0}{ }^{2}}+\frac{1}{(p \xi)^{2}} \\
\frac{1}{\delta_{0}{ }^{2}}=\frac{\pi}{4 R^{2}}=\frac{3 \pi}{2 N} \\
\frac{1}{\xi^{2}}=-3 \ln \left(1-\varphi_{\mathrm{b}}\right)-6 \chi \varphi_{\mathrm{b}}=3 v \varphi_{\mathrm{b}}+\frac{3}{2} \varphi_{\mathrm{b}}{ }^{2}+\ldots
\end{gathered}
$$

All lengths are in units $l$, the monomer length. In eq 2.1, $p$ equals unity for good solvents $(\chi \leq 0.45)$ and 0.81 for a $\Theta$ solvent $(\chi=0.5)$. Equation 2.2 , in which $R=$ $\sqrt{N / 6}$ is the radius of gyration of the polymer, is a wellknown result from the literature. ${ }^{2,8}$ The full logarithmic form of eq 2.3 for $\xi$ was derived in ref 20 . The limiting form $\xi^{-2}=3 v \varphi_{\mathrm{b}}$, where $v=1-2 \chi$ is the Edwards excluded-volume parameter, was introduced by De Gennes $^{22}$ for semidilute solutions in a good solvent. The analogous limit in a $\Theta$ solvent $(\chi=0.5, v=0)$ is $\xi^{-2}=$ $(3 / 2) \varphi_{\mathrm{b}}{ }^{2}$.

Equation 2.1 applies to the case that the concentration profile $\varphi(z)$, where $z$ is the distance from the flat wall, vanishes at the wall: $\varphi(0)=0$. This boundary condition is typical for a continuum model where the segments are treated as points in space; then $\varphi(0)=0$ corresponds to a strongly repelling surface. Since the segments occupy no volume, they can approach the wall to distances smaller than $l$. In a discrete model, like that of Scheutjens and Fleer, ${ }^{16,18}$ the segments occupy a volume $l^{3}$, and the smallest possible distance of approach is $z=0.5$ for their center; the interaction with the wall is expressed through an adsorption energy parameter $\chi_{\mathrm{s}}$, which is $-\infty$ for strong repulsion. In that case the first lattice layer $(0<z<1$, with $z$ also in units $l$ ) is empty, which may be translated to $\varphi(1 / 2)=0$. To get $\varphi(0)=0$ in a lattice model with finite segment size, the wall has to be made weakly repulsive. In ref 20 it was shown that $\varphi(0)=0$ corresponds to $\chi_{\mathrm{s}}=-(1+\chi) / 6$ in flat geometry, as long as $\varphi_{\mathrm{b}}$ is not too high (say $\varphi_{\mathrm{b}} \leq$ $0.15)$. In more concentrated systems the boundary condition becomes concentration dependent, and $\left|\chi_{\mathrm{s}}\right|$ has to increase with $\varphi_{\mathrm{b}}$ to impose $\varphi(0)=0$.

Figure 1 compares eq 2.1 (solid curves) with exact numerical SCF computations (symbols) for $N=400$ and four solvencies $(\chi=0,0.2,0.4$, and 0.5$)$ and the corresponding $\chi_{\mathrm{s}}$ values $(-0.17,-0.20,-0.23$, and -0.25 ) for the lattice model. The agreement is nearly quantitative, apart from some minor deviations in very

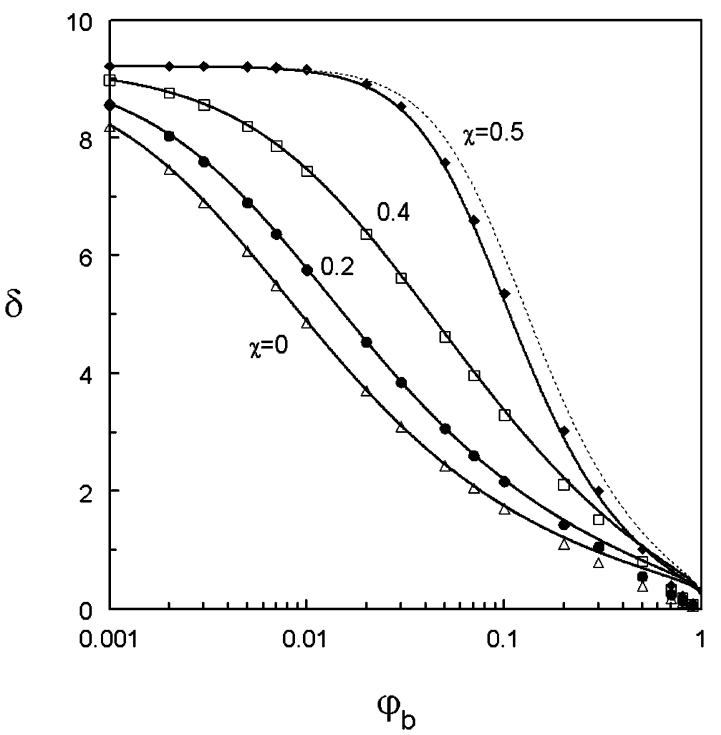

Figure 1. Depletion thickness at a flat wall as a function of the polymer concentration for four solvencies: $\chi=0,0.2,0.4$, and 0.5 . The full curves are given by eq 2.1 , and the data points are SCF lattice results for $\chi_{\mathrm{s}}=-(1+\chi) / 6$. The dotted curve is eq 2.1 with $p=1$ also for $\chi=0.5$. The chain length $N$ is 400 segments $\left(\delta_{0}=9.21 ; R=8.16\right)$.

concentrated systems; here a concentration-dependent boundary condition could possibly improve the agreement. For a $\Theta$ solvent eq 2.1 would overestimate the numerical $\delta$ in the intermediate concentration region when $p$ would be taken as unity (dotted curve in Figure 1); setting $p=0.81$ as discussed above (and in Appendix 1) removes this discrepancy.

2.2. Concentration Profile and Excess Amount. As shown in ref 20, the concentration profile $\varphi(z)$ in a good solvent next to a flat wall with boundary condition $\varphi(0)=0$ is rather accurately given by

$$
\rho(z) \equiv \frac{\varphi(z)}{\varphi_{\mathrm{b}}}=\tanh ^{2}\left(\frac{z}{\delta}\right)
$$

The limiting forms are $\rho(z)=\tanh ^{2}\left(z / \delta_{0}\right)$ in very dilute systems (nearly coinciding with the exact erfc solution ${ }^{2}$ in this limit; see ref 20) and $\rho(z)=\tanh ^{2}(z / \xi)$ in semidilute solutions (which follows from a ground-state approach ${ }^{22}$ ). Integration over this tanh ${ }^{2}$ profile (see also eq 2.6) gives a depletion thickness $\delta$ as defined in eq 2.1 with $p=1$, with limiting forms $\delta_{0}$ (eq 2.2, dilute) and $\xi$ (eq 2.3, semidilute). In Appendix 1 we show that eq 2.4 is also a very good approximation for a $\Theta$ solvent, provided $p=0.81$ is used in eq 2.1.

The depletion zone is a volume $V$ with area $A$ and thickness $\delta$. The excess amount per unit area is given by

$$
-\Gamma=\varphi_{\mathrm{p}} V / A
$$

This equation applies also to spheres (see eq 4.4). In flat geometry VIA may be replaced by $\delta$; hence $-\Gamma=$ $\varphi_{\mathrm{b}} \delta$. The excess amount $\Gamma$ may also be found by integrating over the profile

$$
-\frac{\Gamma}{\varphi_{\mathrm{b}}}=\delta=\int_{0}^{\infty}(1-\rho(z)) \mathrm{d} z
$$

which defines $\delta$ as the zeroth moment of the concentration profile. The limiting behavior of $\Gamma$ is found by 
inserting the limiting forms of eqs 2.2 and 2.3 for $\delta$ and $\xi$ :

$$
\begin{aligned}
& -\Gamma=\varphi_{\mathrm{b}} \delta_{0}(\text { dilute }) \\
& -\Gamma=p \varphi_{\mathrm{b}} \xi= \begin{cases}\left(\varphi_{\mathrm{b}} / 3 v\right)^{1 / 2} & (\text { semidilute good }) \\
\operatorname{atanh}(1 / \sqrt{3}) \approx 0.66 & \text { (semidilute theta) }\end{cases}
\end{aligned}
$$

Plots of $\Gamma$ as a function of $\varphi_{\mathrm{b}}$ will be given later (Figures $6-8)$.

\section{Surface Free Energy}

3.1. Grand Potential. The central quantity we want to calculate is the grand potential $\Omega$ per unit area, which is simply the difference between the surface free energy (or surface tension) $\gamma$ for the surface in contact with the polymer solution and $\gamma_{0}$ for the surface in contact with only solvent:

$$
\Omega=\gamma-\gamma_{0}
$$

We use a dimensionless form for both $\Omega$ and $\gamma$, i.e., units $k T / l^{2}$. In the following paper, ${ }^{21}$ where we consider pair interactions, $\Omega$ and $\gamma$ will become functions of the separation $h$ between two particles. In the present paper we have isolated surfaces $(h=\infty)$, and we abbreviate $\Omega(\infty)$ to a simple $\Omega$.

There are two equivalent ways to calculate $\Omega$ : the osmotic route and the adsorption method.

3.2. Osmotic Route. When the concentration profile is known, $\Omega$ for flat geometry follows from ${ }^{18}$

$$
\Omega=\int_{0}^{\infty} \omega(z) \mathrm{d} z=\int_{0}^{\infty}\left\{\Pi_{\mathrm{b}}-\Pi(z)\right\} \mathrm{d} z
$$

where $\omega$ (in $\left.k T / l^{3}\right)$ is the grand potential density, which is directly coupled to the osmotic pressure profile $\Pi(z)$ (also in $k T / l^{3}$ ). Equation 3.2 is an exact result and involves an integration over space, at fixed bulk osmotic pressure $\Pi_{\mathrm{b}}$, bulk concentration $\varphi_{\mathrm{b}}$, and excess amount $\Gamma$.

In a mean-field picture, $\Pi_{b}$ is given by the FloryHuggins expression: ${ }^{23,24}$

$$
\begin{aligned}
& \Pi_{\mathrm{b}}=-\ln \left(1-\varphi_{\mathrm{b}}\right)-\varphi_{\mathrm{b}}\left(1-\frac{1}{N}\right)-\chi \varphi_{\mathrm{b}}{ }^{2}= \\
& \frac{\varphi_{\mathrm{b}}}{N}+\frac{1}{2} v \varphi_{\mathrm{b}}{ }^{2}+\frac{1}{3} \varphi_{\mathrm{b}}{ }^{3}+\ldots
\end{aligned}
$$

In the expanded version the first term is the ideal (Van't Hoff) contribution, the quadratic term is the dominant contribution for long chains in semidilute solutions in a good solvent, and the cubic term is relevant for semidilute theta conditions.

In the so-called local approximation, which is appropriate for slowly varying profiles, eq 3.3 may also be used for the local osmotic pressure $\Pi(z)$, replacing $\varphi_{\mathrm{b}}$ by the local concentration $\varphi(z)$. In steep gradients, nonlocal contributions involving the second derivative $\mathrm{d}^{2} \varphi / \mathrm{d} z^{2}$ have to be added. ${ }^{25}$ When $\varphi(z)$ and, hence, $\Pi(z)$ are known, $\Omega$ can thus directly be computed from eqs 3.2 and 3.3.

3.3. Adsorption Method. The adsorption method is based upon integration of Gibbs' law

$$
\mathrm{d} \Omega=-\Gamma \mathrm{d} \mu
$$

where $\mu$ (in units $k T$ ) is the chemical potential of the polymer per segment (i.e., the chemical potential per chain is $N \mu$ ). The integration involves a buildup of the system by gradually adding polymer until the desired chemical potential (bulk concentration) is reached. This procedure is completely different from the integration in eq 3.2. Nevertheless, when numerically exact (SF) profiles are used, the results are identical. When analytical approximations are applied, differences may show up, and it is a matter of mathematical convenience which route is followed.

The integration variable $\mu$ in eq 3.4 may be replaced by the integration variable $\Pi$ (osmotic pressure) or $\varphi$ (concentration). During the integration $\Pi$ and $\varphi$ run from zero to their final values $\Pi_{\mathrm{b}}$ and $\varphi_{\mathrm{b}}$, respectively. According to the rule of Gibbs-Duhem, we have $\varphi \mathrm{d} \mu+$ $(1-\varphi) \mathrm{d} \mu_{0}=0$, where $\mu_{0}$ is the solvent chemical potential, related to the osmotic pressure through $\mathrm{d} \mu_{0}$ $=-v_{0} \mathrm{~d} \Pi$, with $v_{0}=l^{3}$ the solvent molecular volume. In dimensionless quantities, we omit the factor $l^{3}$. Replacing $\mathrm{d} \Pi$ by $(\mathrm{d} \Pi / \mathrm{d} \varphi) \mathrm{d} \varphi$, we find

$$
\mathrm{d} \mu=\frac{1-\varphi}{\varphi} \frac{\mathrm{d} \Pi}{\mathrm{d} \varphi} \mathrm{d} \varphi
$$

Here, $\mathrm{d} \Pi / \mathrm{d} \varphi$ is the inverse of the osmotic compressibility. According to eq $3.3,(1-\varphi) \mathrm{d} \Pi / \mathrm{d} \varphi$ is equal to $1 / N$ $+(v-1 / N) \varphi+2 \chi \varphi^{2}$. This expression resembles the equation for $1 / \delta^{2}$ in eqs $2.1-3$. For convenience we rewrite $(1-\varphi) \mathrm{d} \Pi / \mathrm{d} \varphi$ in terms of $1 / \delta_{\mathrm{c}}{ }^{2}$, where $\delta_{\mathrm{c}}$ may be called the "compressibility thickness".

3.4. The "Compressibility Thickness" $\delta_{\mathrm{c}}$. In very dilute solutions $(1-\varphi) \mathrm{d} \Pi / \mathrm{d} \varphi$ equals $1 / N$. From eq 2.2 it is clear that in this limit $1 / \delta_{0}{ }^{2}=(3 \pi / 2) / N$. When we require that $\delta_{\mathrm{c}}$ and $\delta$ have the same limit for (very) low $\varphi$, we have to account for this factor $3 \pi / 2$. Hence we define

$$
\begin{aligned}
& \frac{1}{\delta_{\mathrm{c}}{ }^{2}} \equiv \frac{3 \pi}{2}\left(1-\varphi_{\mathrm{b}}\right) \frac{\mathrm{d} \Pi}{\mathrm{d} \varphi_{\mathrm{b}}}= \\
& \frac{1}{\delta_{0}{ }^{2}}+\frac{3 \pi}{2}\left(v-\frac{1}{N}\right) \varphi_{\mathrm{b}}+3 \pi \chi \varphi_{\mathrm{b}}{ }^{2}
\end{aligned}
$$

Comparison with the expanded form of eq 2.3 shows that only the numerical prefactors of the concentration terms are different. In semidilute solutions in a good solvent the ratio $\delta_{\mathrm{c}} / \delta$ is about $(\pi / 2)^{-1 / 2} \approx 0.80$; for the semidilute theta case this ratio is $\pi^{-1 / 2} / p \approx 0.70$.

Figure 2 illustrates the analogy between $\delta_{\mathrm{c}}\left(\varphi_{\mathrm{b}}\right)$ and $\delta\left(\varphi_{\mathrm{b}}\right)$, for $N=1000$ and $\chi=0,0.4$, and 0.5 . The limiting forms in (very) dilute and (very) concentrated solutions are the same, and in the intermediate concentration range $\delta_{\mathrm{c}}$ is smaller than $\delta$, the more so for poorer solvency. The maximum difference is indeed around $20 \%$ for $\chi=0$ and of order $30 \%$ for $\chi=0.5$.

In terms of $\delta_{\mathrm{c}}$, we may rewrite eq 3.5 as

$$
\mathrm{d} \mu=\frac{2}{3 \pi \delta_{\mathrm{c}}^{2}} \frac{\mathrm{d} \varphi}{\varphi}
$$

Substitution into eq 3.4 and integration gives

$$
\Omega=-\frac{2}{3 \pi} \int_{0}^{\varphi_{\mathrm{b}}} \frac{\Gamma}{\varphi \delta_{\mathrm{c}}^{2}} \mathrm{~d} \varphi
$$

This equation applies to arbitrary geometry. 


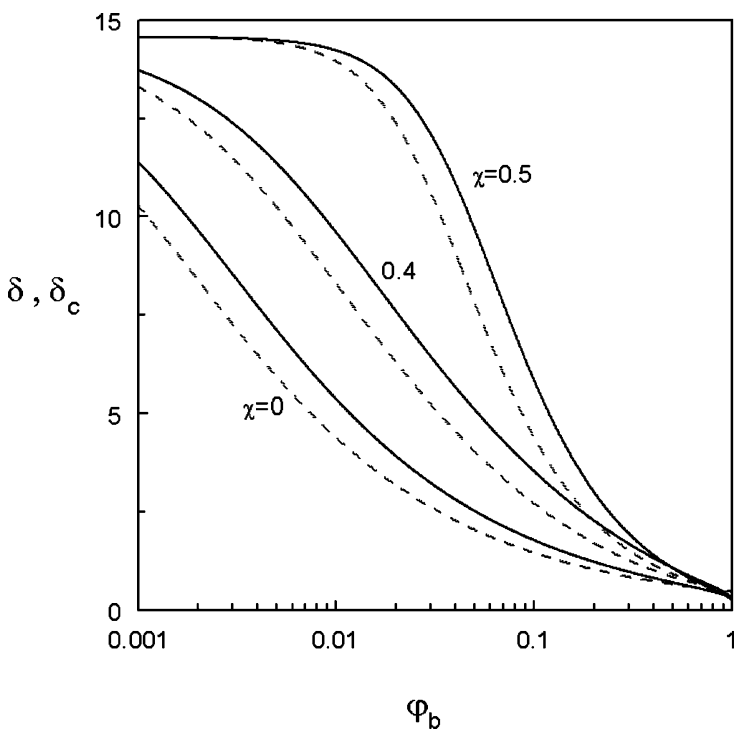

Figure 2. Depletion thickness at a flat wall (eq 2.1, solid curves) and compressibility thickness (eq 3.6, dashed curves) for $N=1000\left(\delta_{0}=14.67\right)$ and three solvencies $(\chi=0,0.4$, and 0.5 ) as a function of the polymer bulk concentration.

3.5. Surface Free Energy for a Plate. For an isolated plate we may substitute $\Gamma / \varphi=-\delta$ (eq 2.6) into eq 3.8 to find

$$
\Omega=\frac{2}{3 \pi} \int_{0}^{\varphi_{\mathrm{b}}} \frac{\delta}{\delta_{\mathrm{c}}^{2}} \mathrm{~d} \varphi
$$

The integral may be solved numerically to find $\Omega$. Results are shown in Figure 3 for $N=1000$ and four values of $\chi$ (solid curves). The symbols in Figure 3 are the exact numerical SCF data with $\chi_{\mathrm{s}}=-(1+\chi) / 6$. It is clear that eq 3.9 provides an excellent description of these lattice results.

Equation 3.9 is analytical but involves a numerical integration. An explicit analytical approximation may be derived from either eq 3.2 (osmotic route) or eq 3.9 (adsorption method). The derivation is given in Appendix 2 . The result is

$$
\Omega=\frac{2 f}{9} \frac{\varphi_{\mathrm{b}}}{\delta} \quad f=1-\left(1-\frac{3}{\pi}\right)\left(\frac{\delta}{\delta_{0}}\right)^{2}
$$

The correction factor $f$ is nearly a constant: its value increases from $3 / \pi=0.955$ in the dilute limit $\left(\delta=\delta_{0}\right)$ to 1 in semidilute solutions. In the dilute limit $(f=3 / \pi)$ eq 3.10 reduces to the exact expression $\Omega=\varphi_{\mathrm{b}} \delta_{0} / N=$ $(2 / 3 \pi) \varphi_{\mathrm{b}} / \delta_{0}$ for ideal chains. ${ }^{26,27}$ Equation 3.10 may be seen as the generalization to finite concentrations.

In semidilute solutions $f$ is unity, and we find the extremely simple result $\Omega=(2 / 9) \varphi_{\mathrm{b}} / \delta$. Clearly, this approximation overestimates $\Omega$ in the dilute limit by a factor $\pi / 3=1.05$. We will see the same effect in the curvature contribution to the excess amount (eq 4.5c) and to the grand potential (eq 4.10) for spheres. In most cases we will be interested in semidilute concentrations, where the simple form of eq 3.10 with $f=1$ is nearly exact, as will be shown below.

For semidilute solutions in a good solvent $\left(\delta \sim \varphi_{\mathrm{b}}{ }^{-1 / 2}\right)$, $\Omega$ in eq 3.10 scales as $\varphi_{\mathrm{b}}{ }^{3 / 2}$. This is nearly the same exponent as in the exact result $\Omega \sim \varphi_{\mathrm{b}}^{1.5426,27}$ in the excluded-volume limit. In the semidilute theta case with $\delta \sim \varphi_{\mathrm{b}}{ }^{-1}$, our scaling result would be $\Omega \sim \varphi_{\mathrm{b}}{ }^{2}$.

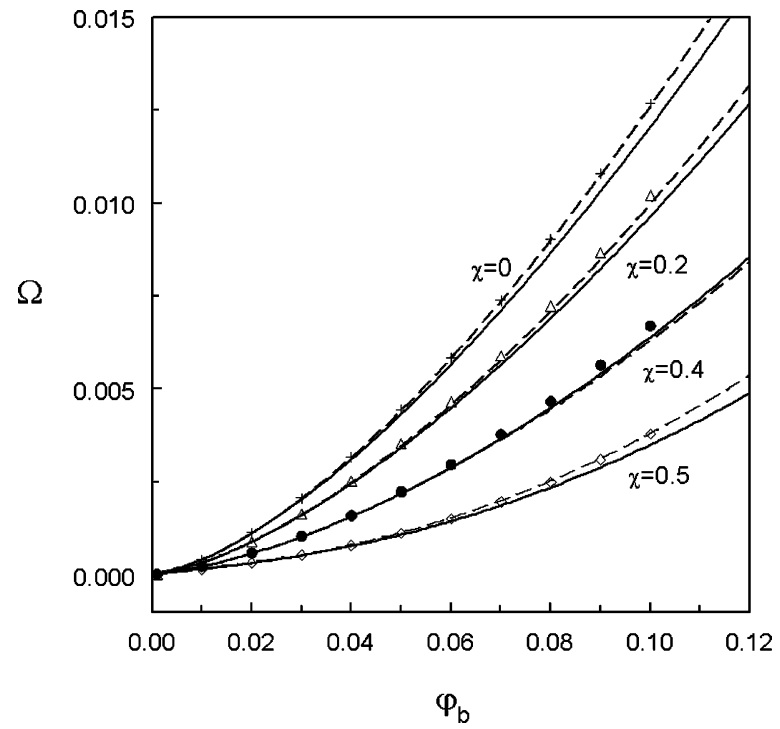

Figure 3. Grand potential for a flat wall in contact with a mean-field polymer solution $(N=1000)$ as a function of the polymer concentration for various solvencies as indicated in the plot: full curves, eq 3.9; dashed curves, eq 3.10; data points, SCF lattice results.

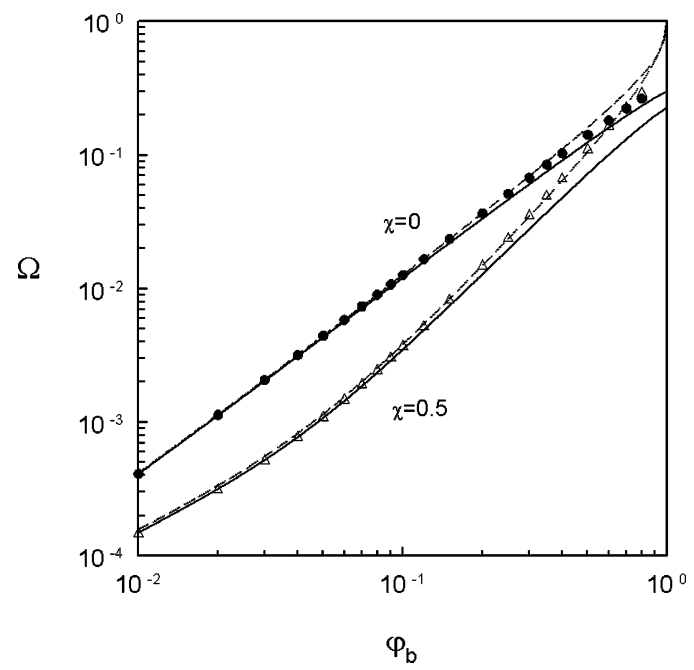

Figure 4. Grand potential for a flat wall in contact with a mean-field polymer solution $(N=1000)$ as a function of the polymer concentration on a double-logarithmic scale for $\chi=0$ and 0.5: full and dashed curves follow eqs 3.9 and 3.10, respectively; data points, SCF lattice results.

The dashed curves in Figure 3 show the quality of the simple expression 3.10. As before, in calculating $\delta$, we used $p=1$ for $\chi=0,0.2$, and 0.4 and $p=0.81$ for $\chi$ $=0.5$. The agreement with the exact SCF data is even better than for eq 3.9 (solid curves) so there is no reason to prefer the numerical integration of eq 3.9 over the simple transparent expression eq 3.10; this equation works very well indeed.

Figure 3 gives results for concentrations up to $\varphi_{\mathrm{b}}=$ 0.1 . It is also of some interest to consider more concentrated systems, where our model is expected to become less accurate. Figure 4 shows such data on a doublelogarithmic scale. The SCF results were calculated with $\chi_{\mathrm{s}}=-(1+\chi) / 6$ throughout. For a concentrated polymer solution this value of $\chi_{\mathrm{s}}$ is not fully consistent with the boundary condition $\varphi(0)=0$ that is the basis of the analytical model. Yet, both eqs 3.9 and 3.10 describe the lattice data quite reasonably up to $\varphi_{\mathrm{b}} \approx 0.5$; for a $\Theta$ 
solvent the simple analytical result of eq 3.10 works again better than the numerical integration of eq 3.9. The scaling law $\Omega \sim \varphi_{\mathrm{b}}{ }^{3 / 2}$ for $\chi=0$ applies to a wide concentration range; for $\chi=0.5 \Omega \sim \varphi_{\mathrm{b}}{ }^{2}$ holds for $\varphi_{\mathrm{b}}$ above around 0.04 . In dilute solutions $\left(\delta=\delta_{0}\right), \Omega$ is linear in $\varphi_{\mathrm{b}}$ for both $\chi=0$ (outside the range shown in Figure 4 ) and $\chi=0.5$ ( $\varphi_{\mathrm{b}}$ of order 0.02 and below). The deviations in very concentrated systems are due to oscillations in the polymer concentration profiles which show up in the exact lattice data; these oscillations are not captured in our simple analytical model. Nevertheless, the SF data seem to follow the scaling laws discussed above also in the concentrated regime.

\section{Spherical Geometry}

4.1. Concentration Profile around a Sphere. As discussed in section 2.2, a rather accurate description for the profile at a flat plate is $\rho=\tanh ^{2}(z / \delta)$. In ref 20 we proposed an extension to spherical geometry

$$
\rho_{\mathrm{s}}=\frac{(z / a+\tanh (z / \delta))^{2}}{(z / a+1)^{2}}
$$

where $a$ is the particle radius. Upon integrating this profile to calculate the excess amount $\Gamma_{\mathrm{s}}$ (see section $4.2)$, the term $\tanh ^{2}(z / \delta)$ gives rise to the "planar" contribution $\Gamma$ to $\Gamma_{\mathrm{s}}$, whereas the double product $(2 z / a)$ $\tanh (z / \delta)$ leads to a "curvature" contribution $\Gamma_{\mathrm{c}}$. Comparison of eq 4.1 with the exact SCF results ${ }^{20}$ showed that this equation is accurate for relatively large particles but underestimates the profile somewhat for small $a$.

We now propose a correction to eq 4.1 which improves the analytical model as to the profile (this section), the excess amount (section 4.2), and the surface free energy (section 4.3). The correction implies a simple replacement of the depletion thickness $\delta$ in the curvature term (the double product in the numerator of eq 4.1) by the "compressibility thickness" $\delta_{\mathrm{c}}$ as defined in eq 3.6 and shown in Figure 2. Hence

$$
\rho_{\mathrm{s}}=\frac{(z / a)^{2}+(2 z / a) \tanh \left(z / \delta_{\mathrm{c}}\right)+\tanh ^{2}(z / \delta)}{(z / a+1)^{2}}
$$

Since $\delta_{\mathrm{c}}$ occurs only in the curvature contribution, the index c could be understood to mean both "compressibility" and "curvature".

The limiting behaviors of eqs 4.1 and 4.2 are identical: $\rho_{\mathrm{s}}=\tanh ^{2}(z / \delta)$ for large $a$ (the planar contribution dominates) and $\rho_{\mathrm{s}}=(1+a / z)^{-2}$ for large $\delta$, which is the Odijk result ${ }^{28}$ for the protein limit. The difference shows up in the intermediate range, where eq 4.2 gives a higher $\rho(z)$ than eq 4.1 because $\delta_{\mathrm{c}}<\delta$ or $\tanh \left(z / \delta_{\mathrm{c}}\right)>$ $\tanh (z / \delta)$.

Figure 5 compares the profiles according to eq 4.1 (dashed curves) and eq 4.2 (solid curves) with numerical SCF data (symbols) for four particles sizes. For the SCF model we have again to choose a value of $\chi_{\mathrm{s}}$ which extrapolates the profile to $\rho_{\mathrm{s}}(0)=0$. For large particle sizes the value $\chi_{\mathrm{s}}=-(\chi+1) / 6$ as used in flat geometry is appropriate, but for small particles curvature effects show up, as discussed in some detail in Appendix 3. The result for spherical geometry is $\chi_{\mathrm{s}}=-(\chi+\Delta \chi) / 6$, where the difference $\Delta \chi$ between the polymer-wall and poly-

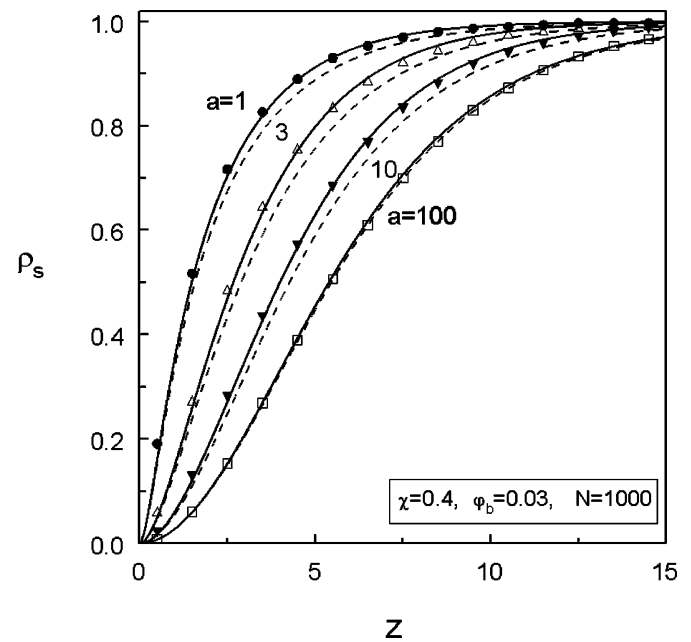

Figure 5. Polymer concentration profiles around a sphere with various sphere radii ( $a=1,3,10$, and 100) in a solution of polymer chains with $\varphi_{\mathrm{b}}=0.03, \chi=0.4$, and $N=1000$. Solid curves follow eq 4.2 and dashed curves eq 4.1. Data points: SCF lattice results with boundary condition as given by eq 4.3 .

mer-solvent Flory-Huggins parameters is a function of the particle radius $a$ according to

$$
\begin{aligned}
& \Delta \chi=\frac{6+2 C^{2}}{(1-C)^{2}} \ln \frac{4+3(1+C)^{2} /(1+2 C)}{6+2 C^{2}} \\
& C \equiv \frac{1}{1+2 a}
\end{aligned}
$$

For $a=1,2$, and 3 eq 4.3 gives $C=1 / 3,1 / 5$, and $1 / 7$ and $\Delta \chi=2.043,1.454$, and 1.267, respectively, whereas for large $a(C \rightarrow 0)$ the "flat limit" $\Delta \chi=6 \ln (7 / 6) \approx 1^{20}$ is obtained.

Figure 5 shows that the agreement between the analytical model of eq 4.2 and the numerical SCF data is nearly quantitative. The improvement with respect to eq 4.1 is not dramatic as to the overall profile, but evaluation of $\Gamma_{\mathrm{S}}$ and $\Omega_{\mathrm{s}}$ requires integration over higher moments of the profile, and then the effect of replacing $\delta$ by $\delta_{\mathrm{c}}$ in the curvature contribution is stronger. We will see in sections 4.2 and 4.3 that this replacement gives good agreement with the exact numerical results for $\Gamma_{\mathrm{s}}$ and $\Omega_{\mathrm{s}}$.

4.2. The Excess Amount around a Sphere. We start from $-\Gamma_{\mathrm{s}}=\varphi_{\mathrm{p}} V / A$, which is eq 2.5 with $\Gamma$ for a flat plate replaced by $\Gamma_{\mathrm{s}}$ for a sphere. When we consider a spherical shell with inner radius $a$ and thickness $\delta_{\mathrm{s}}$ to be void of polymer, we have $V=(4 \pi / 3)\left[\left(a+\delta_{\mathrm{s}}\right)^{3}-\right.$ $\left.a^{3}\right]$ and $A=4 \pi a^{2}$. Hence, eq 2.6 is modified to

$$
\begin{aligned}
-\frac{\Gamma_{\mathrm{s}}}{\varphi_{\mathrm{b}}}=\frac{a}{3}\left[\left(1+\delta_{\mathrm{s}} / a\right)^{3}-1\right] & = \\
& \int_{0}^{\infty}\left(\frac{z}{a}+1\right)^{2}\left(1-\rho_{\mathrm{s}}\right) \mathrm{d} z
\end{aligned}
$$

With eq 4.2 for $\rho_{\mathrm{s}}$ the integrand equals $\left[1-\tanh ^{2}(z / \delta)\right.$ ] $+(2 z / a)\left[1-\tanh \left(z / \delta_{\mathrm{c}}\right)\right]$, which upon integration gives $\delta$ $+\left(\pi^{2} / 12\right) \delta_{\mathrm{c}}{ }^{2} / a$. The first term represents the planar contribution to $-\Gamma_{\mathrm{s}} / \varphi_{\mathrm{b}}$, and the second is the curvature contribution (which vanishes for large $a$ ). Hence

$$
\Gamma_{\mathrm{s}}=\Gamma+\Gamma_{\mathrm{c}} ;-\Gamma=\varphi_{\mathrm{b}} \delta ;-\Gamma_{\mathrm{c}}=\frac{\pi^{2}}{12} \varphi_{\mathrm{b}} \frac{\delta_{\mathrm{c}}^{2}}{a}
$$




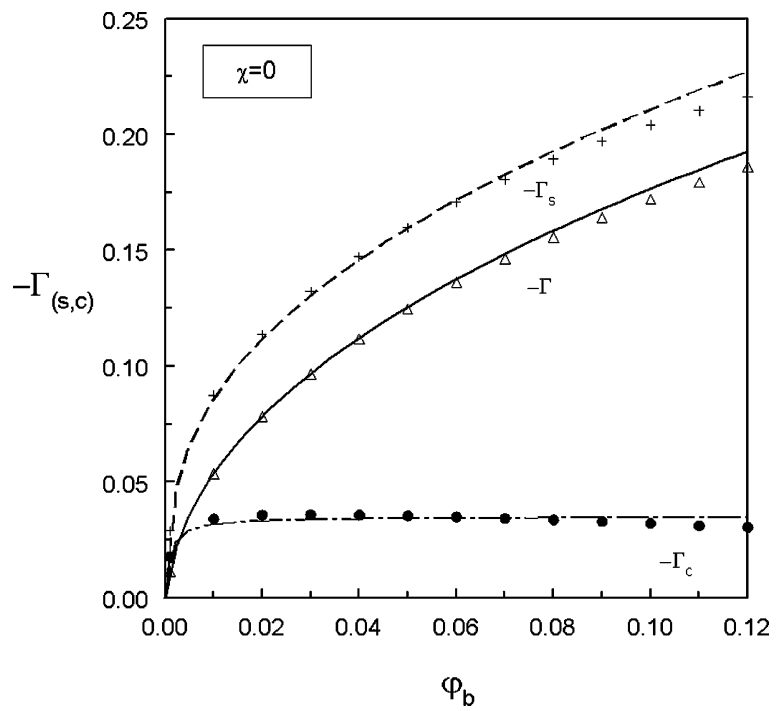

Figure 6. Excess amount of polymer segments around a sphere with $a=5$ for $N=1000$ and $\chi=0$ as a function of the polymer concentration. The overall $\left(\Gamma_{\mathrm{s}}\right)$, flat $(\Gamma)$, and curvature $\left(\Gamma_{\mathrm{c}}\right)$ contributions are plotted separately. Solid curve, eq $4.5 \mathrm{~b}$; dot-dashed curve, eq 4.5c; dashed curve, eq 4.5a. Data points: SCF lattice results with boundary condition as in eq 4.3 .

In the dilute limit eq 4.5, which is based upon the tanh ${ }^{2}$ profile, reduces to $-\Gamma_{\mathrm{s}} / \varphi_{\mathrm{b}}=\delta_{0}+\pi^{2} \delta_{0}^{2} / 12 a$. For this dilute limit an exact result, derived from the exact profile in terms of complementary error functions, is available: Aarts et al. ${ }^{8}$ obtained $-\Gamma_{\mathrm{s}} / \varphi_{\mathrm{b}}=\delta_{0}+\pi \delta_{0}{ }^{2} / 4 a$. The difference is just a factor $\pi / 3=1.05$ in the curvature term. We encountered the same factor in the dilute limit of eq 3.10 for $\Omega$ (and we will meet it again in its curvature contribution $\Omega_{c}$; see eq 4.10). Since in most cases we will be interested in relatively concentrated solutions where the tanh ${ }^{2}$ profile is adequate, we stick to the factor $\pi^{2} / 12$ in eq $4.5 \mathrm{c}$. We have to realize, however, that this result is $5 \%$ too high for very dilute systems. When desired, we could correct for this small effect by multiplying eq $4.5 \mathrm{c}$ by the correction factor $f$ as defined in eq 3.10.

Upon combining the second and third parts of eq 4.4, we find an expression for the thickness $\delta_{\mathrm{s}}$ of the depletion zone around a sphere:

$$
\left(1+\frac{\delta_{\mathrm{s}}}{a}\right)^{3}=1+3 \frac{\delta}{a}+\frac{\pi^{2}}{4} \frac{\delta_{\mathrm{c}}^{2}}{a^{2}}
$$

Equation 4.5 is in nice agreement with the numerical SCF data, as shown in Figures $6-8$, which give $\Gamma_{\mathrm{s}}$ and its planar $(\Gamma)$ and curvature $\left(\Gamma_{\mathrm{c}}\right)$ contributions as a function of $\varphi_{\mathrm{b}}$ for $N=1000, a=5$, and $\chi=0$ (Figure 6), $\chi=0.4$ (Figure 7), and $\chi=0.5$ (Figure 8). In the SCF computations, $\Gamma_{\mathrm{c}}$ was obtained as the difference between $\Gamma_{\mathrm{s}}$ (calculated for a sphere) and $\Gamma$ (calculated for a plate), and the boundary condition as given by eq 4.3 was applied.

The planar contribution $-\Gamma$ increases monotonically with the polymer concentration, and eq $4.5 \mathrm{~b}$ describes the numerical data quite well. In all cases $\Gamma\left(\varphi_{\mathrm{b}}\right)$ is linear in (very) dilute solutions, according to eq $2.7 \mathrm{a}$. In semidilute solutions in a good solvent $-\Gamma$ increases as $\left(\varphi_{\mathrm{b}} / 3 v\right)^{1 / 2}$, and in $\Theta$ solvents a plateau $-\Gamma=p \sqrt{2 / 3}=$ $\operatorname{atanh}(1 / \sqrt{3}) \approx 0.66$ is found at (very) high concentrations (see eq 2.7b).

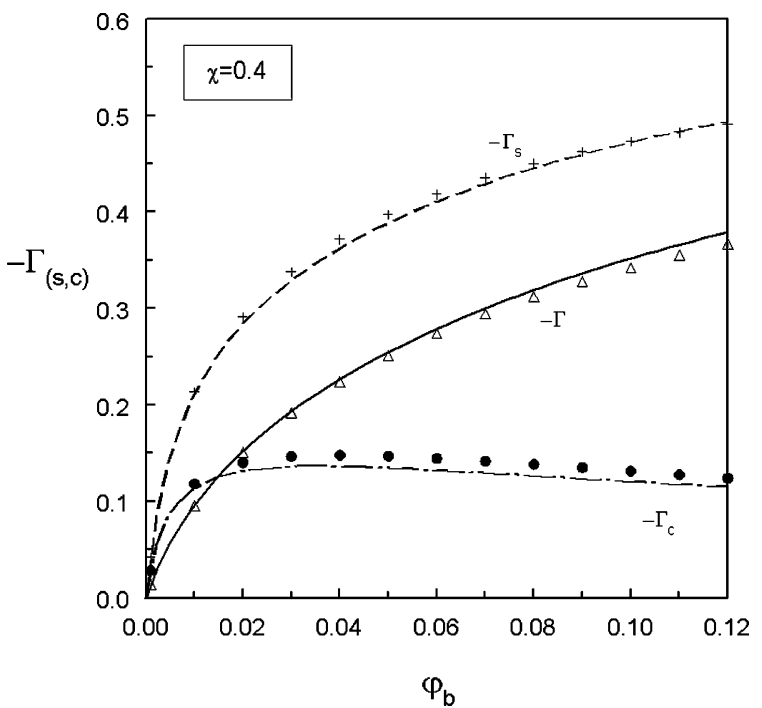

Figure 7. As Figure 7 but for $\chi=0.4$.

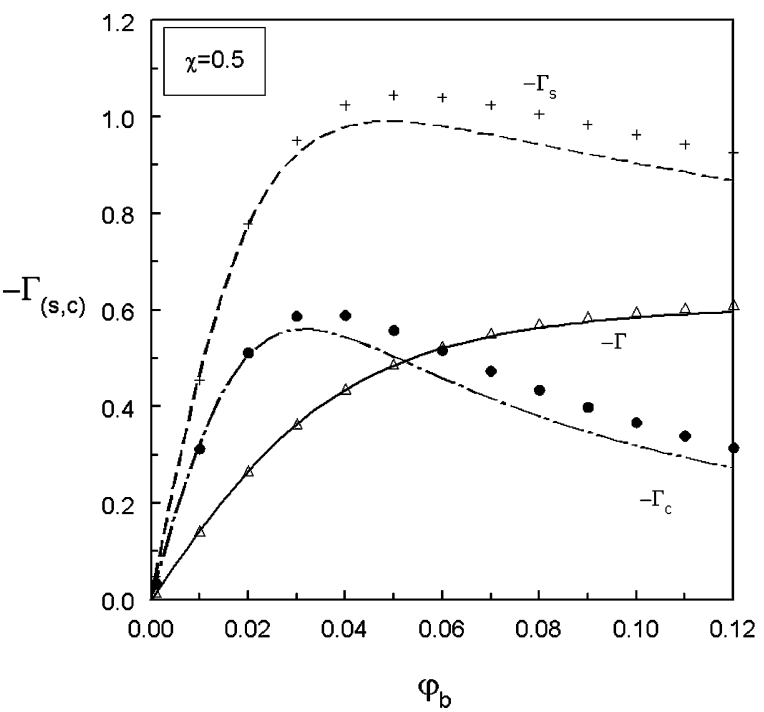

Figure 8. As Figures 7 and 8 but for $\chi=0.5$.

The curvature contribution $\Gamma_{\mathrm{c}}$ is always linear in concentration for dilute solutions, reaches quickly a plateau for $\chi=0$, and passes through a maximum for higher $\chi$. These findings are easily rationalized by inserting the appropriate limiting forms for $\delta_{\mathrm{c}}$ into eq 4.5c. In dilute solutions eq 3.6 gives $\delta_{0}{ }^{2}=\delta_{\mathrm{c}}{ }^{2}=2 N / 3 \pi$, and $\delta_{\mathrm{c}}{ }^{-2}=(3 \pi / 2)\left(v \varphi_{\mathrm{b}}+2 \chi \varphi_{\mathrm{b}}{ }^{2}\right)$ in the semidilute case where the $1 / N$ terms vanish. Hence

$$
\begin{aligned}
& -\Gamma_{\mathrm{c}}=\frac{N}{6 a} \varphi_{\mathrm{b}}(\text { dilute }) \\
& \qquad-\Gamma_{\mathrm{c}}=\frac{\pi}{18 a} \frac{1}{v+2 \chi \varphi_{\mathrm{b}}} \text { (semidilute) }
\end{aligned}
$$

The dilute limit describes the initial linear part; in this case we applied eq $4.5 \mathrm{c}$ with the factor $\pi / 4$ instead of $\pi^{2} / 12$. The semidilute asymptote shows that indeed a plateau $-\Gamma_{\mathrm{c}}=\pi / 18 a$ is reached for $\chi=0(v=1)$, whereas $-\Gamma_{\mathrm{c}}$ is inversely proportional to $a \varphi_{\mathrm{b}}$ for high $\varphi_{\mathrm{b}}$ and $\chi=$ $0.5(v=0)$. The maximum $\left|\Gamma_{\max }\right|$ of $\left|\Gamma_{\mathrm{c}}\right|$ is found from $\mathrm{d}\left(1 / \Gamma_{\mathrm{c}}\right) / \mathrm{d} \varphi_{\mathrm{b}}=0$ with the full expression (eq 3.6) for $\delta_{\mathrm{c}}{ }^{-2}$ :

$$
\varphi_{\max }=\frac{1}{\sqrt{2 N \chi}} ; \quad-\frac{1}{\Gamma_{\max }}=\frac{18 a}{\pi}\left(v-\frac{1}{N}+4 \chi \varphi_{\max }\right)
$$


The fact that $-\Gamma_{\mathrm{c}}$ passes through a maximum may also be explained in a physical picture. In (very) dilute solutions an increase in $\varphi_{\mathrm{b}}$ increases the negative excess at a constant (high) depletion thickness $\delta_{0}$. With increasing concentration in a very good solvent $(\chi=0)$, the depletion thickness decreases at a rate $\left(\delta \sim \varphi_{\mathrm{b}}{ }^{-1 / 2}\right)$ that just compensates the increase of $\varphi_{\mathrm{b}}: \Gamma_{\mathrm{c}} \sim \delta^{2} / \varphi_{\mathrm{b}}$ remains constant. For poorer solvents the decrease of $\delta$ is faster and the colloid limit of small $\delta / a$ (where the curvature contribution becomes small) is approached. For semidilute theta conditions $-\Gamma_{\mathrm{c}}$ decreases as $\varphi_{\mathrm{b}}{ }^{-1}$ : $-\Gamma_{\mathrm{c}}=\pi\left(18 a \varphi_{\mathrm{b}}\right)^{-1}$.

4.3. Grand Potential around a Sphere. In this case the analytical integration in the adsorption route turns out to be very easy. Upon inserting $\Gamma_{\mathrm{s}}=\Gamma+\Gamma_{\mathrm{c}}$ from eq 4.5 into eq 3.8, we immediately see that $\Omega_{\mathrm{s}}$ can be separated into two terms:

$$
\Omega_{\mathrm{s}}=\Omega+\Omega_{\mathrm{c}}
$$

The planar contribution $\Omega$ is given by eq 3.9 (which requires a numerical integration) or the easier explicit approximation eq 3.10. Since we have analyzed this planar contribution in some detail in section 3.5 , we concentrate here only on the curvature part $\Omega_{\mathrm{c}}$.

This curvature term becomes very simple because $\Gamma_{\mathrm{c}} d \varphi$ $\sim \delta_{\mathrm{c}}{ }^{2}$ (eq 4.5c), so that the integrand $\left(\Gamma_{\mathrm{d}} / \varphi\right) \delta_{\mathrm{c}}{ }^{-2}$ in the curvature part of eq 3.8 becomes concentration-independent! We directly end up with

$$
\Omega_{\mathrm{c}}=\frac{\pi}{18} \frac{\varphi_{\mathrm{b}}}{a}
$$

In fact, it was this simple result, which-as shown below-agrees very well with the exact numerical SCF data for concentrations up to $\varphi_{\mathrm{b}} \simeq 0.1$ that set us on the track to replace $\delta$ by $\delta_{\mathrm{c}}$ in the curvature contribution of the profile (see eq 4.2 and Figure 5). Equation 4.10 can also be derived from the osmotic route; details are given in Appendix 4.

We note that the exact result derived by Louis et al. ${ }^{26}$ in the dilute limit is $\Omega_{\mathrm{c}}=\varphi_{\mathrm{b}} / 6 a$, which is again lower by a factor $\pi / 3$. As before, we could multiply eq 4.10 by the correction factor $f$ defined in eq 3.10; in most cases we will just take $f=1$. The surprising feature of eq 4.10 is that essentially the "dilute" result holds up to segment volume fractions of order $10 \%$. This is demonstrated in Figure 9, which gives a plot of $\Omega_{\mathrm{c}}\left(\varphi_{\mathrm{b}}\right)$ for $N=1000$ (corresponding to $\delta_{0}=14.6$ ) and $a=5$ for two solvencies: $\chi=0$ and $\chi=0.5$. The straight line in this figure, drawn according to eq 4.10, describes the exact data (symbols) rather accurately up to $\varphi_{\mathrm{b}} \approx 0.10$ in a good solvent and up to slightly lower concentrations in a $\Theta$ solvent. For higher concentrations deviations are seen, with the exact results (calculated with eq 4.3 for $\chi_{\mathrm{s}}=$ $-(\chi+\Delta \chi) / 6)$ bending downward in a good solvent and upward in a $\Theta$ solvent. Part of these deviations may be due to the fact that at high concentrations the boundary condition $\varphi(0)=0$, which is the basis of all our analytical approximations, requires a concentration (and solvency)dependent $\chi_{\mathrm{s}}$. More importantly, as remarked in the discussion of Figure 4, oscillations in the profile show up in the exact results for concentrated solutions. Therefore, we do not try to extend the analytical model to this case and are satisfied that our model in its simplest form works well up to $\varphi_{\mathrm{b}} \approx 0.1$.

In Figure 10 we check the dependence of $\Omega_{\mathrm{c}}$ on curvature $1 / a$ for $\varphi_{\mathrm{b}}=0.05, N=1000$, and two

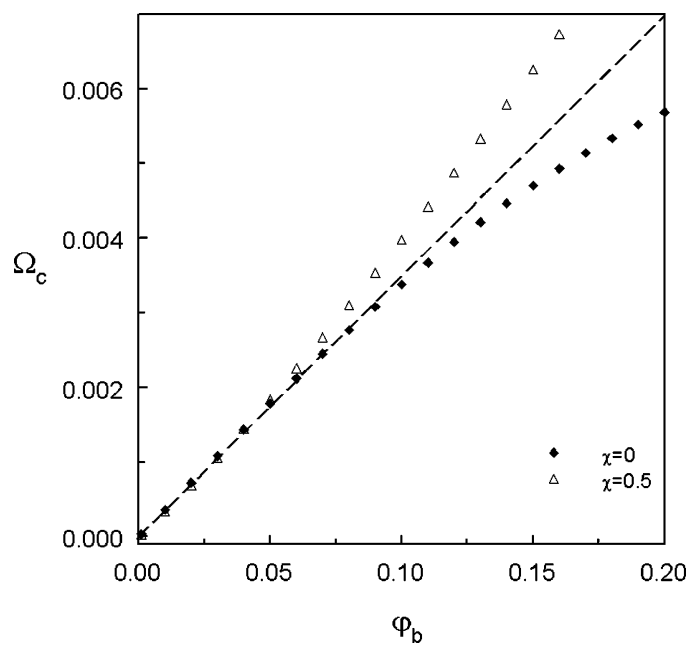

Figure 9. Curvature contribution to the grand potential at a spherical particle as a function of $\varphi_{\mathrm{b}}$, for $a=5, \chi=0$ and 0.5 , and $N=1000$. Dashed line: eq 4.10; data points: SCF lattice results with boundary condition as in eq 4.3.

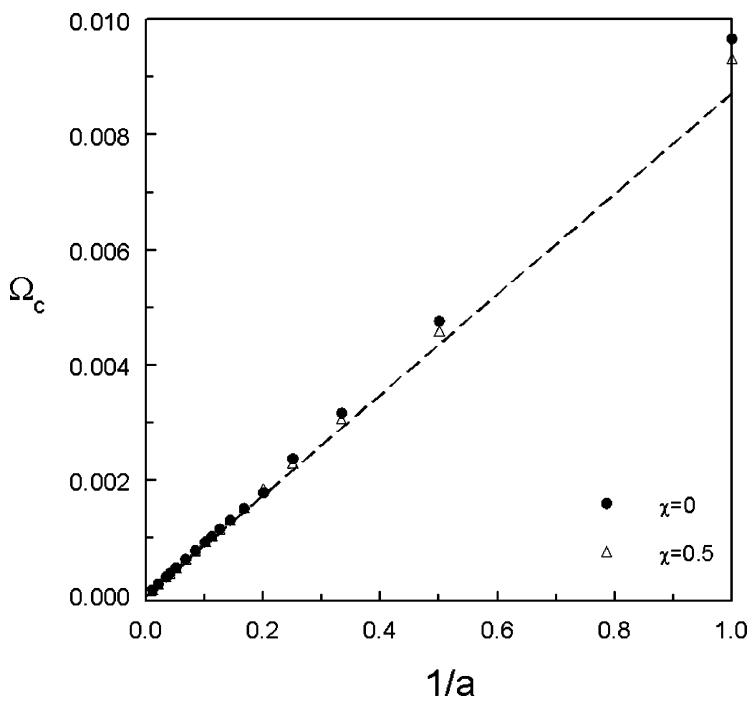

Figure 10. Curvature contribution to the grand potential at a spherical particle as a function of curvature $1 / a$, for $\varphi_{\mathrm{b}}=$ $0.05, \chi=0$ and 0.5, and $N=1000$. Dashed line: eq 4.10; data points: SCF lattice results with boundary condition as in eq 4.3 .

solvencies. The agreement of eq 4.10 with the exact data is excellent, down to rather small particles. Only for $a$ $=1$ (the smallest particle size that can be handled in the lattice model) is a small deviation visible.

The ratio $\Omega_{c} / \Omega$, which is a measure for the relative importance of the curvature contribution, equals $(\pi / 4) \delta /$ $a$. Since $\delta$ depends on concentration, this ratio does as well. In relatively concentrated solutions (small $\delta$ ), the colloid limit is approached and the contribution of $\Omega_{\mathrm{c}}$ is small (unless the particles are quite small).

Finally, in Figure 11 we show the $\varphi_{\mathrm{b}}$ dependence of the total grand potential $\Omega_{\mathrm{s}}$ of a sphere, which is the sum of the planar contribution $\Omega$ and the curvature term $\Omega_{\mathrm{c}}$, for $\chi=0.5, N=1000$, and four values of the particle radius $a$. Symbols are the SCF results with eq 4.3 for $\Delta \chi$. The curves represent $\Omega_{\mathrm{s}}=\Omega+\Omega_{\mathrm{c}}$, using eq 3.10 for the planar contribution $\Omega$ and eq 4.10 for $\Omega_{\text {c }}$. The planar contribution $\Omega$ was plotted separately in Figure 3. The curves in Figure 11 give an excellent description of the lattice results for small polymer concentrations and relatively large spheres, and even 


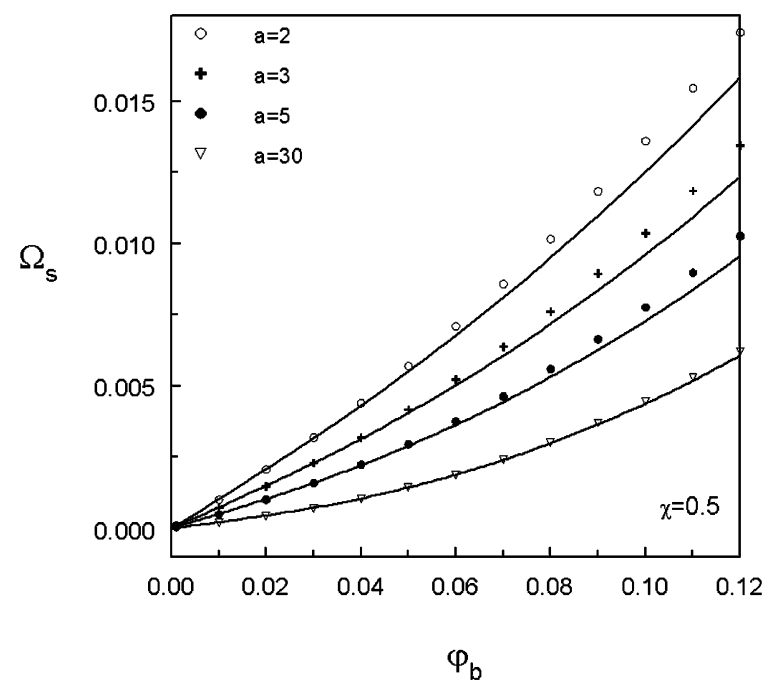

Figure 11. Grand potential $\Omega_{\mathrm{s}}$ of a spherical particle in contact with a mean-field polymer solution $(N=1000)$ as a function of the polymer concentration, for $\chi=0.5$ and various sphere radii as indicated. Solid curves: analytical results for $\Omega_{\mathrm{S}}$ (the sum of eqs 3.10 and 4.10 ); data points: SCF lattice results with boundary condition as in eq 4.3.

for higher concentrations and smaller particles the agreement is fair. Hence, the simple analytical expressions 3.10 and 4.10 capture the main effects of the grand potential around a sphere. We note that Figure 11 applies to a $\Theta$ solvent, where the curvature contribution $\Omega_{\mathrm{s}}$ (which does not depend on solvency) is relatively important because the planar contribution $\Omega$ (which does depend on solvency) is small (see Figure 3). For better solvents a diagram like Figure 11 is closer to Figure 3: the planar contribution dominates.

\section{Concluding Remarks}

We obtained analytical equations for the excess amount and the grand potential (or surface free energy) near a surface in a solution containing nonadsorbing polymer, both for flat and for spherical geometry. We started from a recent mean-field equation for the concentration profile and depletion thickness $\delta$ at a flat wall as a function of chain length $N$, polymer concentration $\varphi_{\mathrm{b}}$, and solvency $\chi$. The polymer excess amount, which is negative for depletion, follows from integration over the concentration profile. For spheres, we improved a previously proposed equation for the concentration profile around a sphere, using not only the "flat" depletion thickness $\delta$ but also a "curvature" thickness $\delta_{c}$ which in semidilute solutions is slightly smaller than $\delta$. The results for the adsorbed amount agree quite well with numerical lattice results over the entire solvency range $0 \leq \chi \leq 0.5$.

We also derived analytical expressions for the grand potential (surface free energy), using both the adsorption method and the osmotic route. The grand potential for flat geometry turns out to be proportional to $\varphi_{\mathrm{b}} / \delta$. For spheres of radius $a$, an additional curvature term $(\pi / 18) \varphi_{\mathrm{b}} / a$ enters. These results are rather accurate up to a polymer segment volume fraction of $10 \%$ and even give a reasonable description for more concentrated systems.

The present study is a suitable starting point for the pair interaction between colloidal particles in a solution containing nonadsorbing chains, as described in the following paper. ${ }^{21}$

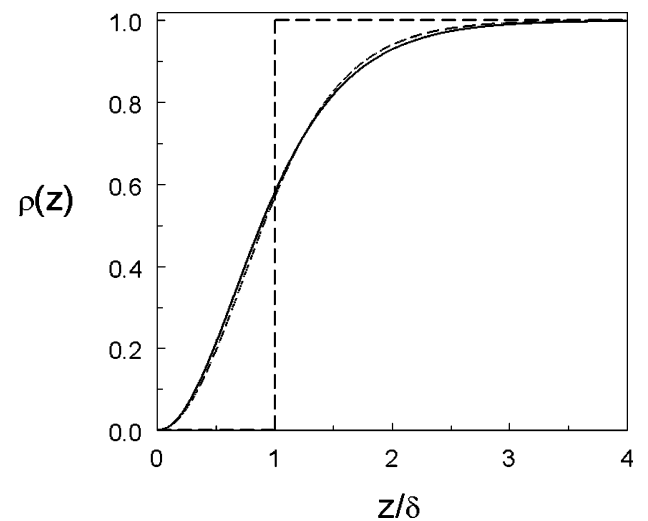

Figure 12. Normalized polymer concentration profile as a function of the normalized distance $z / \delta$ from a flat plate according to eq 2.4 with $\delta=p \xi$ (solid curve) and eq A1 with $\delta$ $=\xi$ (dashed curve). The step function at $z=\delta$ is also indicated; it has the same zeroth moment as the two continuous profiles.

\section{Appendix 1. Concentration Profile in a $\Theta$ Solvent}

In the dilute limit, the concentration profile in a $\Theta$ solvent is the same as in a good solvent and is given by eq 2.4. We note that our mean-field model does not capture the chain swelling in a good solvent, which makes $R$ in a good solvent larger than in a $\Theta$ solvent. However, the ground-state solution for semidilute theta conditions is different: 20

$$
\rho(z)=\frac{\cosh (\sqrt{8} z / \xi)-1}{\cosh (\sqrt{8} z / \xi)+2}
$$

Integration over this profile gives a depletion thickness $p \xi$, with $p=\sqrt{3 / 2} \operatorname{atanh}(1 / \sqrt{3}) \approx 0.81 .^{20}$

In this paper eq $\mathrm{A} 1$ is not used because the simpler $\tanh ^{2}$ form of eq 2.4 is a good approximation also in a $\Theta$ solvent, provided we correct for the different width of the profile. To that end we replace $\delta$ in eq 2.4 for a semidilute solution by $p \xi$. Figure 12 shows that the theta profile of eq A1 (dashed curve) nearly coincides with $\tanh ^{2}[z /(p \xi)]$ (solid curve), and the zeroth moment of both profiles (determining the excess) is exactly the same. This justifies eq 2.1 and provides an excellent approximation of the excess amount under most conditions. Only for solvencies slightly better than $\chi=0.5$ do we expect that the "step function" for $p(0.81$ for $\chi=$ $0.5,1$ for $\chi<0.5)$ is slightly inaccurate.

\section{Appendix 2. Derivation of Eq 3.10}

We insert the expanded form (eq 3.3b) for $\Pi(z)$ and $\Pi_{\mathrm{b}}$ into eq 3.2 , using the profile $\varphi(z)=\varphi_{\mathrm{b}} \tanh ^{2}(z / \delta)(\mathrm{eq}$ 2.4). Upon integration of the three separate terms we get

$$
\begin{aligned}
& \Omega=\varphi_{\mathrm{b}} \delta\left[\frac{1}{N}+\frac{2}{3} v \varphi_{\mathrm{b}}\right.\left.+\frac{23}{45} \varphi_{\mathrm{b}}^{2}\right]= \\
& \frac{2}{9} \varphi_{\mathrm{b}} \delta\left[\frac{3}{\pi} \frac{1}{\delta_{0}{ }^{2}}+3 v \varphi_{\mathrm{b}}+\frac{23}{10} \varphi_{\mathrm{b}}{ }^{2}\right]
\end{aligned}
$$

In the second form we replaced $N$ by $(3 \pi / 2) \delta_{0}{ }^{2}$ (eq 2.2). The last two terms in this second version may be compared with $(p \xi)^{-2}=3 v \varphi_{\mathrm{b}}+\left(3 / 2 p^{2}\right) \varphi_{\mathrm{b}}{ }^{2}$ according to eq 2.3. In the linear term of this expression, which vanishes for a $\Theta$ solvent, $p$ may be taken unity. The quadratic term is hardly relevant for a good solvent, and 
in a $\Theta$ solvent the numerical coefficient is $3 / 2 p^{2}=2.306$, which is very close to $23 / 10$. Hence, to a very good approximation we may write the last factor of eq A2 as $(3 / \pi) \delta_{0}-2+(p \xi)^{-2}=\delta^{-2}-(1-3 / \pi) \delta_{0}^{-2}$, where we used $(p \xi)^{-2}=\delta^{-2}-\delta_{0}^{-2}$ (eq 2.1). This gives eq 3.10 of the main text.

Essentially the same result may be derived from the adsorption route (eq 3.9), which involves a completely different type of integration. In the dilute regime $\delta=$ $\delta_{\mathrm{c}}=\delta_{0}$, and the integral of eq 3.9 reduces to $\varphi_{\mathrm{b}} / \delta_{0}$ so that $\Omega=(2 / 3 \pi) \varphi_{\mathrm{b}} / \delta_{0}$, which is the dilute limit of eq 3.10 . For the semidilute regime we take the limiting expressions for $\delta$ and $\delta_{\mathrm{c}}$. In semidilute solutions in a good solvent $\delta=(3 v \varphi)^{-1 / 2}$ and $\delta_{\mathrm{c}}{ }^{-2}=(3 \pi v / 2) \varphi$; the integral of eq 3.9 gives now $(\pi / 3)(3 v)^{1 / 2} \varphi_{\mathrm{b}}{ }^{3 / 2}=(\pi / 3) \varphi_{\mathrm{b}} / \delta$ so that $\Omega=(2 / 9) \varphi_{\mathrm{b}} / \delta$, which is the semidilute version of eq 3.10 . Similarly, for semidilute theta conditions we have $\delta=$ $\left(2 p^{2} / 3\right)^{1 / 2} \varphi^{-1}$ and $\delta_{c}{ }^{-2}=(3 \pi / 2) \varphi^{2}$, which leads to an integral $(3 \pi / 4)\left(2 p^{2} / 3\right)^{1 / 2} \varphi_{\mathrm{b}}{ }^{2}=\left(\pi p^{2} / 2\right) \varphi / \delta$ and to $\Omega=\left(p^{2 /}\right.$ 3) $\varphi_{\mathrm{b}} / \delta$; the prefactor $p^{2} / 3=0.217$ is very close to $2 / 9=$ 0.222 .

These results illustrate that the osmotic and adsorption procedures, though following entirely different paths, are equivalent as to the final outcome.

\section{Appendix 3. Lattice Boundary Condition for a Sphere}

In the appendix of ref 20 we derived the value of $\chi_{\mathrm{s}}$ which has to be used in a planar lattice in order to find a concentration profile with boundary condition $\varphi(0)=$ 0 . The starting point is the lattice recurrency relation for the layer next to the surface. For the other layers, this recurrency relation is the discrete version of the Edwards equation. ${ }^{17}$

For the present purpose, we rewrite eqs A3a,b of ref 20 as

$$
\lambda_{0}+\lambda_{+} g_{2} / g_{1}=\mathrm{e}^{\lambda_{-} \Delta \chi}
$$

We used the ground-state approximation by replacing the end-point distribution function $G_{i, N}$ for layer $i$ by the ground-state eigenfunction $g_{i} \sim \sqrt{\rho_{i}}=\tanh \left(z_{i} / \delta\right)$, and we omitted the concentration terms in the field for the layer adjacent to the wall $(i=1)$, where the polymer concentration is low in the case of depletion. So, the only contribution to the field experienced by a segment in layer 1 is due to contacts with the wall and with solvent; those with other segments are neglected. The parameter $\Delta \chi \equiv \chi_{\mathrm{PS}}-\chi$ measures the difference in contact energy of a polymer segment with the surface $\left(\chi_{\mathrm{PS}} \equiv-6 \chi_{\mathrm{s}}\right)$ and with the solvent $(\chi)$. The $\lambda$ 's in eq A3 are lattice parameters, with $\lambda_{+}$and $\lambda_{-}$representing outward and inward steps, respectively, and $\lambda_{0}$ refers to steps within the same layer. In a planar six-choice cubic lattice as used before $\lambda_{+}=\lambda_{-}=1 / 6$ and $\lambda_{0}=1-\lambda_{+}-\lambda_{-}=4 / 6$.

With $g_{1}=\tanh (0.5 / \delta) \approx 0.5 / \delta$ and $g_{2}=\tanh (1.5 / \delta) \approx$ $1.5 / \delta$, eq A3 for the planar case reduces to $(4+3) / 6=$ $\mathrm{e}^{\Delta \chi / 6}$ or $\Delta \chi=6 \ln (7 / 6) \approx 1$. In the main text of this paper we translated $\Delta \chi=1$ as $\chi_{\mathrm{PS}}=1+\chi$ or $\chi_{\mathrm{s}}=-(1+\chi) / 6$.

For a spherical lattice the transition probabilities become a function of the layer number. ${ }^{18,29}$ When $r$ is the radial distance from the center of the lattice to the middle of a given shell, we have $\lambda_{-}=A_{-} / 6 L$ and $\lambda_{+}=$ $A_{+} / 6 L$, where $A_{-}=4 \pi(r-1 / 2)^{2}$ and $A_{+}=4 \pi(r+1 / 2)^{2}$ are the inner and outer areas of each shell, respectively, and $L=(4 \pi / 3)\left[(r+1 / 2)^{3}-(r-1 / 2)^{3}\right]=4 \pi\left(r^{2}+1 / 12\right)$ is the number of sites in this shell. Hence

$$
\lambda_{-}=\frac{(r-1 / 2)^{2}}{6 r^{2}+1 / 2} ; \quad \lambda_{+}=\frac{(r+1 / 2)^{2}}{6 r^{2}+1 / 2} ; \quad \lambda_{0}=\frac{4 r^{2}}{6 r^{2}+1 / 2}
$$

For large $r$ these equations reduce to $\lambda_{+}=\lambda_{-}=1 / 6$ and $\lambda_{0}=4 / 6$. For small $r, \lambda_{+}>\lambda_{-}$since outward steps have a higher a priori probability than inward ones.

We may define a curvature parameter $C=1 / 2 r$, which takes the values $1 / 1,1 / 3,1 / 5,1 / 7 \ldots$ for the inner four (...) shells in the lattice. We rewrite eq $\mathrm{A} 4$ as

$$
\lambda_{-}=\frac{1}{6} \frac{(1-C)^{2}}{1+C^{2} / 3} ; \quad \lambda_{+}=\frac{1}{6} \frac{(1+C)^{2}}{1+C^{2} / 3} ; \quad \lambda_{0}=\frac{4 / 6}{1+C^{2} / 3}
$$

In eq A3 we need these parameters for the first solution shell, with its center at $r=a+1 / 2$ and corresponding curvature parameter $C=1 /(1+2 a)$. For example, for $a$ $=1$ we have $\lambda_{+}=4 / 14, \lambda_{-}=1 / 14, \lambda_{0}=9 / 14$, for $a=2$ $\lambda_{+}=9 / 38, \lambda_{-}=4 / 38, \lambda_{0}=25 / 38$, and for $a=3$ the result is $\lambda_{+}=16 / 74, \lambda_{-}=9 / 74, \lambda_{0}=49 / 74$.

To find $\Delta \chi$ from eq A3, we need the ratio $g_{2} / g_{1}$. In the spirit of the ground-state approximation, we use again $g_{i} \sim \sqrt{\rho_{i}}$. With the simplest form (eq 4.1) for $\rho_{i}$, using $\tanh \left(z_{i} / \delta\right) \approx z_{i} / \delta$, we find with $z_{1}=0.5$ and $z_{2}=1.5$

$$
\frac{g_{2}}{g_{1}}=\frac{3+6 a}{3+2 a}=\frac{3}{1+2 C}
$$

In the second version we have used again $C=1 /(1+$ $2 a$ ). For large $a$ this equation gives $g_{2} / g_{1}=3$, as for the planar case. By substituting eqs A5 and A6 into eq A3, eq 4.3 for $\Delta \chi$ in the main text is obtained.

\section{Appendix 4. Derivation of Eq 4.10 from the Osmotic Route}

The generalization of eq 3.2 to spherical geometry is

$$
\Omega_{\mathrm{s}}=\frac{1}{a^{2}} \int_{0}^{\infty}(z+a)^{2}\left\{\Pi_{\mathrm{b}}-\Pi(z)\right\} \mathrm{d} z
$$

which is an exact mean-field result. Using the local approximation (i.e., eq 3.3 also for $\Pi(z)$ ) and with the expanded form $\Pi=\varphi / N+v \varphi^{2} / 2+\varphi^{2} / 3$, we can insert the profile $\varphi=\varphi_{\mathrm{b}} \rho_{\mathrm{s}}$ with $\rho_{\mathrm{s}}$ from eq 4.2 into eq $\mathrm{A} 7$. The linear term gives an integrand $\left(\varphi_{\mathrm{b}} / N\right)(z / a+1)^{2}\left(1-\rho_{\mathrm{s}}\right)$ $=\left(\varphi_{\mathrm{b}} / N\right)\left\{\left[1-\tanh ^{2}(z / \delta)\right]+(2 z / a)\left(1-\tanh \left(z / \delta_{\mathrm{c}}\right)\right]\right\}$, showing again a planar and a curvature contribution. Here we consider only the curvature part, leading to $\Omega_{\mathrm{c}}=\left(\varphi_{\mathrm{b}} / N\right)\left(\pi^{2} / 12 a\right) \delta_{\mathrm{c}}{ }^{2}$. In very dilute solutions, where this linear part is the leading term, $\delta_{\mathrm{c}}{ }^{2}$ equals $2 N / 3 \pi$ so that $\Omega_{\mathrm{c}}=\pi \varphi_{\mathrm{b}} / 18 a$ (the exact solution is lower by a factor $\pi / 3=1.05)$. For the quadratic term we get the integrand $(v / 2) \varphi_{\mathrm{b}}{ }^{2}(z / a+1)^{2}\left(1-\rho_{\mathrm{s}}{ }^{2}\right)$. With $\rho_{\mathrm{s}}$ from eq 4.2 there is no analytical integral, but we may approximate $1-\rho_{\mathrm{s}}{ }^{2}$ $=\left(1+\rho_{\mathrm{s}}\right)\left(1-\rho_{\mathrm{s}}\right)$ as $2\left(1-\rho_{\mathrm{s}}\right)$ over most of the integration range. Then this quadratic term gives for the curvature part $\Omega_{\mathrm{c}}=v \varphi_{\mathrm{b}}{ }^{2}\left(\pi^{2} / 12 a\right) \delta_{\mathrm{c}}{ }^{2}$, which with $\delta_{\mathrm{c}}{ }^{2}$ $=2 /\left(3 \pi v \varphi_{\mathrm{b}}\right)$ for the semidilute good-solvent regime leads again to $\Omega_{\mathrm{c}}=\pi \varphi_{\mathrm{b}} / 18 \mathrm{a}$. Similarly, the cubic term gives $(1 / 3) \varphi_{\mathrm{b}}{ }^{3}(z / a+1)^{2}\left(1-\rho_{\mathrm{s}}{ }^{3}\right)$, where $\left(1-\rho_{\mathrm{s}}{ }^{3}\right)=\left(1-\rho_{\mathrm{s}}\right)(1+$ $\left.\rho_{\mathrm{s}}+\rho_{\mathrm{s}}^{2}\right)$ may be approximated as $3\left(1-\rho_{\mathrm{s}}\right)$. Hence, its contribution to $\Omega_{\mathrm{c}}$ is $\varphi_{\mathrm{b}}{ }^{3}\left(\pi^{2} / 12 a\right) \delta_{\mathrm{c}}{ }^{2}$. With $\delta_{\mathrm{c}}{ }^{2}=2 /\left(3 \pi \varphi_{\mathrm{b}}{ }^{2}\right)$ under semidilute theta conditions the result is once more $\Omega_{\mathrm{c}}=\pi \varphi_{\mathrm{b}} / 18 a$. Indeed, numerical integration of eq A7 (with the logarithmic form for П) shows that for $\varphi_{\mathrm{b}}$ 
$<0.1 \Omega_{\mathrm{c}}=\Omega_{\mathrm{s}}(a)-\Omega_{\mathrm{s}}(\infty)$ does not depend on $\chi$ and is linear in $\varphi_{\mathrm{b}}$, with a slope which is close to $\pi / 18 a$.

\section{References and Notes}

(1) Some recent reviews: Poon, W. C. K. J. Phys.: Condens. Matter 2002, 14, R859. Tuinier, R.; Rieger, J.; de Kruif, C. G. Adv. Colloid Interface Sci. 2003, 103, 1.

(2) Eisenriegler, E. J. Chem. Phys. 1983, 79, 1052.

(3) Eisenriegler, E.; Hanke, A.; Dietrich, S. Phys. Rev. E 1996 54,1134

(4) Asakura, S.; Oosawa, F. J. Polym. Sci. 1958, 33, 183.

(5) Vrij, A. Pure Appl. Chem. 1976, 48, 471.

(6) Fuchs, M.; Schweizer, K. S. Europhys. Lett. 2000, 51, 621.

(7) Fuchs, M.; Schweizer, K. S. J. Phys.: Condens. Matter 2002 , 14, R239.

(8) Aarts, D. G. A. L.; Tuinier, R.; Lekkerkerker, H. N. W. J. Phys.: Condens. Matter 2002, 14, 7551

(9) Schmidt, M.; Denton, A. R.; Brader, J. M. J. Chem. Phys. 2003, 118, 1541.

(10) Bolhuis, P. G.; Louis, A. A.; Hansen, J. P.; Meijer, E. J. J. Chem. Phys. 2001, 114, 4296.

(11) Bolhuis, P. G.; Louis, A. A.; Hansen, J.-P. Phys. Rev. Lett. 2002, 89, 128302.

(12) Bolhuis, P. G.; Louis, A. A.; Meijer, E. J. Phys. Rev. Lett. 2003, 90, 068304.

(13) Sear, R. P. Phys. Rev. E 2002, 66, 051401.

(14) Chatterjee, A. P.; Schweizer, K. S. Macromolecules 1999, 32, 923.
(15) Chen, Y.-L.; Schweizer, K. S.; Fuchs, M. J. Chem. Phys. 2003 , $118,3880$.

(16) Scheutjens, J. M. H. M.; Fleer, G. J. J. Phys. Chem. 1979, 83,1619 .

(17) Edwards, S. F.; Freed, K. F. J. Phys. A 1969, 2, 145.

(18) Fleer, G. J.; Cohen Stuart, M. A.; Scheutjens, J. M. H. M.; Cosgrove, T.; Vincent, B. Polymers at Interfaces; Chapman \& Hall: London, 1993

(19) Fleer, G. J.; van Male, J.; Johner, A. Macromolecules 1999, $32,825,845$.

(20) Fleer, G. J.; Skvortsov, A. M.; Tuinier, R. Macromolecules 2003, 36, 7857.

(21) Tuinier, R.; Fleer, G. J. Macromolecules 2004, 37, 8764.

(22) de Gennes, P. G. C. R. Acad. Sci. B. 1980, 290, 509. See also: Scaling Concepts in Polymer Physics; Cornell University Press: Ithaca, NY, 1979; Chapter 9.

(23) Flory, P. J. J. Chem. Phys. 1941, 9, 660; Principles of Polymer Chemistry; Cornell University Press: Ithaca, NY, 1953.

(24) Huggins, M. L. J. Chem. Phys. 1941, 9, 440.

(25) Hong, K. M.; Noolandi, J. Macromolecules 1981, 14, 727.

(26) Louis, A. A.; Bolhuis, P. G.; Meijer, E. J.; Hansen, J.-P. J. Chem. Phys. 2002, 116, 10547.

(27) Hanke, A.; Eisenriegler, E.; Dietrich, S. Phys. Rev. E 1999 , 59,6853 .

(28) Odijk, T. Macromolecules 1996, 29, 1842

(29) Van der Schoot, P. P. A. M.; Leermakers, F. A. M. Macromolecules 1988, 21, 1867.

MA0485742 